\title{
Review Article \\ Digging Up the Human Genome: Current Progress in Deciphering Adverse Drug Reactions
}

\author{
Shih-Chi Su, ${ }^{1}$ Wen-Hung Chung, ${ }^{1,2}$ and Shuen-Iu Hung ${ }^{3}$ \\ ${ }^{1}$ Department of Dermatology, Drug Hypersensitivity Clinical and Research Center, Chang Gung Memorial Hospital, \\ 199 Tung-Hwa North Road, Taipei 105, Taiwan \\ ${ }^{2}$ College of Medicine, Chang Gung University, 259 Wen-Hua First Road, Taoyuan 333, Taiwan \\ ${ }^{3}$ Institute of Pharmacology, School of Medicine, Infection and Immunity Research Center, VYM Genome Research Center, \\ National Yang-Ming University, No. 155, Section 2, Linong Street, Taipei 112, Taiwan
}

Correspondence should be addressed to Wen-Hung Chung; chung1@cgmh.org.tw and Shuen-Iu Hung; sihung@ym.edu.tw

Received 13 December 2013; Accepted 3 February 2014; Published 10 March 2014

Academic Editor: Wei Chiao Chang

Copyright (C) 2014 Shih-Chi Su et al. This is an open access article distributed under the Creative Commons Attribution License, which permits unrestricted use, distribution, and reproduction in any medium, provided the original work is properly cited.

\begin{abstract}
Adverse drug reactions (ADRs) are a major clinical problem. In addition to their clinical impact on human health, there is an enormous cost associated with ADRs in health care and pharmaceutical industry. Increasing studies revealed that genetic variants can determine the susceptibility of individuals to ADRs. The development of modern genomic technologies has led to a tremendous advancement of improving the drug safety and efficacy and minimizing the ADRs. This review will discuss the pharmacogenomic techniques used to unveil the determinants of ADRs and summarize the current progresses concerning the identification of biomarkers for ADRs, with a focus on genetic variants for genes encoding drug-metabolizing enzymes, drugtransporter proteins, and human leukocyte antigen (HLA). The knowledge gained from these cutting-edge findings will form the basis for better prediction and management for ADRs, ultimately making the medicine personalized.
\end{abstract}

\section{Introduction}

Adverse drug reactions (ADRs) are side effects occurring within the approved dosage and labeling recommendations. Severe ADRs, which require hospitalization, are a significant clinical problem in drug therapy because they can be permanently disabling or result in death. The incidence of severe ADRs has been estimated at $6.2-6.7 \%$ in hospitalized patients and the incidence of fatal ADRs is estimated to be $0.15-0.3 \%$ [1]. From a clinical aspect, ADRs can be broadly divided into two types, type $\mathrm{A}$ and type $\mathrm{B}$ [2]. Type $\mathrm{A}$ reactions are considered as a magnification of a drug's therapeutic effect and represent the majority of ADRs. This type of condition is predictable from the known pharmacology of a drug and typically dose dependent. By contract, type $\mathrm{B}$ reactions are less common and do not involve the pharmacological effects of a drug. Moreover, most individuals are not susceptible to type B ADRs, which are, thus, being termed "idiosyncratic." With the advance of the current understanding in their underlying mechanisms, some type B reactions now become potentially avoidable although totally unpredictable in the past.

In addition to the impact on health care, ADR remains a huge cost burden for pharmaceutical industry. It has been reported that 56 out of 548 newly approved drugs in the US either had to be withdrawn from the market or achieved a black box warning due to adverse reactions that were unpredicted by clinical trials from 1975 to 1999 [3]. Although controversial, there is an estimate that the cost of bringing a single new drug to market is US\$802 million [4]. Thus, severe ADRs pose tremendous challenges both to patient care and to pharmaceutical development. The current successes in discovering specific genotypes that are highly associated with certain ADRs are encouraging; however, a more comprehensive understanding is essential for dealing with this complex problem. In this review, we discuss the pharmacogenomic techniques used to explore the pathogenesis of ADRs and summarize the current progresses concerning 
genetic associations and predictors for the occurrence of ADRs, with a focus on genetic variants for genes encoding drug-metabolizing enzymes, drug-transporter proteins, and human leukocyte antigen (HLA).

\section{Pharmacogenomic Strategies for Studying ADR}

In the past few decades, many genes which are implicated in simple, monogenic disorders have been discovered by using linkage analysis and positional cloning approaches. However, these methods were less successful in mapping genes that are involved in complex diseases, like ADRs, because such diseases typically are caused by several genes, each with a portion of overall contribution. Researchers, thus, began to conduct the association studies using the candidate-gene approach to search for the statistical correlation between genetic variants and a disease. These genetic association studies, through which the relation of selected genes/genotypes with the etiological role of a disease in a group of populationbased samples from affected and unaffected (case versus control) individuals was analyzed, are likely to be more useful than linkage studies for studying complex traits because they can have greater statistical power to find numerous genes of small effect [5]. In spite of its advantage, it has been reported that the association studies of the same disease using such candidate-gene approach are often inconsistent in their findings and that the first study to report an association often presents a stronger effect than that observed in subsequent studies [6].

With the completion of the human genome project [7] and the availability of comprehensive data on variability in human genome from the HapMap [8], huge strides have been made in our understanding of single nucleotide polymorphism (SNP) and the impact of interindividual genetic variants on the risk of complex diseases. These findings together with the development of modern methods and techniques allowing the prosecution of large-scale association studies have evolved the studies of complex disorders from the candidate-gene approach to the genomewide association study (GWAS). Unlike the candidate-gene approach that highlighted the selected genes, GWAS aims to analyze the genotype of SNPs throughout the whole genome, not simply focusing on those that are obvious candidates for effects on the disease of interest. Due to this open nature, GWAS does not require an initial hypothesis for exploring the genetic predisposing factors to a complex disease. However, a limitation of GWAS is that a large sample size is required to discover the SNPs with relatively low odds ratios [9]. This often harnesses the studies on severe, idiosyncratic ADRs which occur at very low frequencies, unless samples are collected via international collaboration [10].

In addition, advances in DNA sequencing technologies that allow substantial increases in sequencing content while dramatically decreasing the cost per base have facilitated the advent of high-throughput sequencing methods, often referred to as next-generation sequencing (NGS). These techniques, including whole-genome sequencing that reads the complete sequence of an individual's genome at a single time and whole-exome sequencing that captures only the parts of the DNA which code for proteins, have been successfully applied to numerous disease-targeted tests in disease diagnostics [11]. The central advantage of NGS over GWAS on exploration of genetic etiology of polygenic diseases is that NGS can directly identify the causal variants whereas GWAS primarily is designed for seeking markers that are intended to represent causal variation indirectly. Furthermore, these sequencing-based methods possess higher explorative power than does GWAS, enabling to discover the causal variations with low allele frequencies $(<5 \%)$ in complex traits [12], although the development of chip-based genotyping advances greatly. Yet, until recently, studies of uncovering the genetic susceptibility to complex diseases or severe ADRs using NGS techniques are still very limited.

\section{Drug-Metabolizing Enzymes}

Interindividual differences in drug disposition have been recognized as important and common causes of adverse drug reactions [13]. Drug metabolism is generally classified into two phases, termed phase I and phase II. Phase I reactions encompass oxidation or reduction reactions, usually through the actions of cytochrome 450 oxidative enzymes or reductases. These phase I drug-metabolizing enzymes (DME) process the parent drugs for phase II reactions by creating a conjugation site on the drug. Subsequently, phase II DME acts to conjugate a hydrophilic entity onto the intermediate product, allowing the formation of a more polar metabolite that can be excreted in the urine or bile [14]. Genetic variants of DME genes such as SNP, insertion/deletion, and gene duplication may alter either the expression level or the functional activity of an enzyme, resulting in aberrant pharmacokinetics and ultimately leading to ADR. With the substantial progress in current pharmacogenomic studies, numerous genetic variants of DME have been identified as predisposing factors to ADRs. A comprehensive review that covers the genetic variations in phase I and phase II DMEs to the safety and toxicity of drug therapy has been published recently [15]. Here, we summarize only "known valid" DME biomarkers and their effects on drug safety.

The majority of DMEs belong to the CYP gene superfamily, which encodes a phase I enzyme family, the cytochrome p450 superfamily [16]. Polymorphisms in CYP1A2, CYP2C9, CYP2C19, and CYP2D6 have been evaluated to contribute to clinically significant differences in exposure to several drugs [17]. Among these SNPs, several CYP2C9 variants (predominantly CYP2C $9 * 2$ and $* 3$ alleles) are relevant to adverse effects of numerous antiepileptics, antidepressants, nonsteroidal anti-inflammatory agents, sulfonylurea antidiabetic drugs and, most critically, oral anticoagulants (e.g., acenocoumarol and warfarin) [18]. Myriads of clinical studies have shown that the CYP2C9 polymorphism should be considered in warfarin therapy [19]. Similar information is known for another member of CYP2C subfamily, CYP2C19. The CYP2C19*2 allele was associated 
with a marked decrease in platelet responsiveness to clopidogrel, an anticoagulant [20] while the pharmacokinetics of citalopram, an antidepressant, were influenced by the CYP2C19*2 and CYP2C19*17 alleles [21, 22]. Dose adjustments for these drugs based on CYP2C19 genotypes have been suggested. In addition, CYP2D6, another most extensively studied polymorphic, CYP, is involved in the metabolism of a large number of drugs, such as antiarrhythmics, tricyclic and second-generation antidepressants, antipsychotics, $\beta$-blockers, opioid analgesics, and anticancer drugs [23]. Carriers of duplicated variants of CYP2D6 (CYP2D6*2) have been shown to be susceptible to the ADR of codeine treatment $[24,25]$. Cumulative pharmacokinetic data from patients and healthy volunteers have also suggested a reduction in drug dosage for several antidepressants based on CYP2D6 phenotypes [25].

Furthermore, genetic polymorphisms of phase II DMEs are also known to influence the drug metabolism and the development of ADRs. An association of the genetic variation in the promoter region of uridine diphosphate glucuronosyltransferase 1A1 gene (UGT1A1*28) with irinotecan-associated toxicity has been prescribed $[26,27]$. The wild-type allele of UGT1A1 has six TA repeats in the promoter region while the UGT1Al $* 28$ has seven TA repeats, producing an enzyme with reduced activity [26]. Another notable example is the involvement of thiopurine S-methyltransferase (TPMT) allelic variants (predominantly $\mathrm{TPMT} * 2, \mathrm{TPMT} * 3 \mathrm{~A}$, and $\mathrm{TPMT} * 3 \mathrm{C}$ ) in mercaptopurine- or azathioprine-related adverse events [28-30]. Other phase II DMEs whose genetic polymorphisms have been correlated with drug toxicity are $N$-acetyltransferase type I (NAT1) and type II (NAT2) [31]. By comparison with the NAT2 genes, only a small number of NAT1 variants result in alteration of phenotypes. An increased incidence of drug toxicities in subjects carrying polymorphic NAT2 alleles has been reported when received hydralazine and sulfasalazine [32-34].

\section{Drug-Transporter Proteins}

Drug-transporter proteins (DTPs) represent another group of important determinants that govern the pharmacokinetics. These transporters are integral membrane proteins that mediate the influx or efflux transport of drug metabolites across the membrane using active and passive mechanisms [35]. Influx DTPs are mainly composed of the solute carrier (SLC) superfamily, including the organic cation transporters (OCTs), the multidrug and toxin extrusion (MATE) transporters, the organic anion transporters (OATs), and the organic anion transporting polypeptides (OATPs), while efflux transporters consist of members of the ATP-binding cassette $(A B C)$ superfamily, such as P-glycoprotein (P-gp/MDR1), breast cancer resistance protein (BCRP), and transporters of the multidrug resistance-associated protein (MRP) family. For a more detailed description regarding the impact of DTPs on drug efficacy and toxicity, refer to a recent comprehensive review [36]. Here, we highlight those with welldefined pharmacogenomic roles in the development of ADRs.

OATP1B1, encoded by SLCO1B1, remains one of the most extensively studied influx DTPs, owing to the prevalence of clinically relevant polymorphisms [37]. A wellcharacterized SLCO1B1 variant is the loss-of-function polymorphism c.521T $>C$ (rs4149056). The genetic association of rs4149056 with myopathy induced by simvastatin, a 3-hydroxy-3-methylglutaryl-coenzyme (HMG-CoA) reductase inhibitor used for controlling elevated cholesterol, has been identified $[38,39]$. It is, thus, recommended that genetic tests of SLCO1B1 genotypes may be clinically useful tools for preventing simvastatin-induced muscle toxicity [40]. Similar finding was also observed in the OCTs, whose expressions and activities are crucial for the delivery of antineoplastics to the target tissues. A SNP of OCT2 gene (SLC22A2), rs316019, was found to be associated with reduced nephrotoxicity from cisplatin in cancer patients [41]. This observation was supported by the pharmacokinetic study of cisplatin in OCT2 knockout mice. In addition, another group of influx DTPs that moves small organic anions against their concentration gradient using a $\mathrm{Na}+$ gradient is the OAT family. Of particular significance in drug disposition are OAT1 and OAT3, encoded by SLC22A6 and SLC22A8, respectively. A SNP in the intergenic region between SLC22A6 and SLC22A8 (rs10792367) was recently identified to be associated with hypertension to hydrochlorothiazide [42], although association studies of genetic variants in genes encoding OATs with changes in drug disposition are very limited.

Polymorphisms in efflux transporters are also known to be involved in the toxicity to drug treatment or predisposition to ADRs. A noteworthy example is the pharmacogenomic finding regarding P-glycoprotein (ABCB1/MDR1), the first human $A B C$ transporter gene formerly characterized through its ability to confer a multidrug resistant (MDR) phenotype to certain chemotherapy drugs in cancer cells [43]. Among numerous variants of $A B C B 1$ identified, a correlation of the ABCB1 3435T>C (rs1045642) was observed with cyclosporine-induced nephrotoxicity [44, 45]. In addition, functional effects of genetic variants in the ABCB1 gene have been considered as haplotypes rather than independent SNPs, as the use of $\mathrm{ABCB} 1$ haplotypes has been applied to predict the pharmacokinetics of many drugs [46-48]. Other lines of evidence also indicate that the SNPs of another ABC gene, ABCC4 (encoding MRP4), showed an association with ADRs induced by cyclophosphamide and methotrexate in cancer patients $[49,50]$. A brief summary of the association between genetic variations involved in pharmacokinetics and pharmacodynamics and their related ADRs is shown in Table 1.

\section{Human Leukocyte Antigen (HLA)}

Other than genes involved in pharmacokinetics and pharmacodynamics, an immune etiology has been suggested for 
TABLE 1: Associations between genetic variants involved in pharmacokinetics and pharmacodynamics and their related ADRs.

\begin{tabular}{|c|c|c|c|}
\hline Genetic variants & ADR & Drug & Reference \\
\hline $\mathrm{ABCB} 1$ (rs1045642) & Nephrotoxicity & Cyclosporine & {$[44,45]$} \\
\hline ABCC4 (rs9561778) & Leukopenia/toxicity & Cyclophosphamide & {$[49]$} \\
\hline $\mathrm{CYP} 2 \mathrm{C} 19 * 2$ & Decreased platelet responsiveness & Clopidogrel & {$[20]$} \\
\hline CYP2C19 $* 2$, CYP2C19*17 & Altered pharmacokinetics & Citalopram & {$[21,22]$} \\
\hline $\mathrm{CYP} 2 \mathrm{D} 6 * 2$ & Opioid intoxication & Codeine & {$[24]$} \\
\hline Polymorphic NAT2 & Toxicity & Hydralazine, sulfasalazine & {$[32-34]$} \\
\hline SLC22A2 (rs316019) & Reduced nephrotoxicity & Cisplatin & {$[41]$} \\
\hline SLCO1B1 (rs4149056) & Myopathy & Simvastatin & {$[38,39]$} \\
\hline TPMT $* 2$, TPMT $* 3 \mathrm{~A}$, TPMT $* 3 \mathrm{C}$ & Hematologic toxicity & Mercaptopurine, azathioprine & {$[30]$} \\
\hline $\mathrm{UGT} 1 \mathrm{~A} 1 * 28$ & Toxicity & Irinotecan & {$[26,27]$} \\
\hline
\end{tabular}

ABCB1: ATP-binding cassette subfamily B member 1; ABCC4: ATP-binding cassette subfamily C member 4; CYP: cytochrome p450 superfamily; NAT2: $N$ acetyltransferase type II; SLC22A2: solute carrier family 22 member 2; SLCO1B1: solute carrier organic anion transporter family member 1B1; TPMT: thiopurine S-methyltransferase; UGT1A1: uridine diphosphate glucuronosyltransferase 1A1.

a great number of $\mathrm{ADRs}$, in particular, type $\mathrm{B}$ reactions [76]. Many attempts to search for the associations with specific HLAs have been made, and the findings often are drug and ethnicity specific as summarized in Table 2. Such type of ADRs is recognized as drug-induced hypersensitivity reactions that involves major histocompatibility- (MHC-) restricted drug presentation and subsequent activation of specific immune responses. Two types of the classical MHC molecules mediate this process: the MHC class I molecules, expressed by most nucleated cells, and the MHC class II molecules, expressed by specialized antigen-presenting cells (APCs). In humans, the classical MHC class I molecule is encoded by three loci known as HLA-A, HLA-B, and HLA-C; the classical MHC class II molecule is encoded by three loci known as HLA-DR, HLA-DQ, and HLADP. MHC class I and class II molecules may regulate the drug hypersensitivity by presenting antigenic drugs to $\mathrm{CD} 8+$ (cytotoxic) and CD4+ (helper or regulatory) $\mathrm{T}$ cells, respectively. Because drugs are usually too small to likely trigger an immunogenic response, several mechanistic models, including the hapten/prohapten model, the $\mathrm{p}-\mathrm{i}$ model, and the altered repertoire model, have been proposed to explain how small molecular synthetic compounds are recognized by $\mathrm{T}$ cells in an $\mathrm{MHC}$-dependent/independent fashion. The hapten/prohapten concept proposes that the drug or its metabolite (hapten/prohapten) reacts with a selfprotein through covalent binding to generate a haptenated, de novo product. This product then undergoes antigen processing to create a novel MHC ligand that is loaded onto the MHC and trafficked to the cell surface, where it activates antigen-specific T lymphocytes $[77,78]$. In addition, a second concept, the $\mathrm{p}-\mathrm{i}$ (pharmacological interaction with immune receptors) model, describes that a noncovalent, labile interaction of the drug with the MHC receptor at the cell surface is involved in MHC-dependent/independent T-cell stimulation by various drugs [79]. Neither cellular metabolism nor antigen processing is required in such an interaction. This model, to some extent, explains certain cases where drug hypersensitivity occurs rapidly, since the immunogenic complexes produced by drug presentation are unlikely to depend on antigen processing and cellular metabolism. Another concept, the altered repertoire model, has recently been proposed, according to which the drugs or its metabolites can bind noncovalently within the pocket of the peptide binding groove of certain MHC molecules with extraordinary specificity, allowing a new repertoire of endogenous self-peptides to be bound and presented. This concept is supported by the findings from various studies of abacavir-mediated drug hypersensitivity that the binding of abacavir to the antigen-binding cleft of HLA-B $* 5701$ sterically hindered the binding of the original repertoire of peptides, thereby prompting the binding of a new repertoire of peptides bearing immunogenic neoepitopes [80-82].

The striking examples of HLA associations with ADRs are HLA-B $* 1502$ with carbamazepine-induced Stevens-Johnson syndrome (SJS) and toxic epidermal necrolysis (TEN) in many regions of Southeast Asia [59-61], HLA-B*5801 with allopurinol-induced SJS/TEN/hypersensitivity syndrome (HSS) [53-55], and HLA-B*5701 with abacavir-induced hypersensitivity syndrome in the Caucasian population $[51,52]$. These HLA-linked ADRs typically occur in defined populations owing to the prevalence of the specific alleles. The knowledge gained from such pharmacogenomic studies has led to a further development of genetic tests for identifying individuals at risk of these serious conditions [76]. Moreover, regardless of the diversity of genetic backgrounds and the difference in sample sizes examined, other HLA-drug associations that contribute to the pathogenesis of ADRs have been reported: HLA-A $* 3101$ and HLA-B $* 1511$ with carbamazepine-induced HSS $[63,65,66,83]$, HLA-B $* 1301$ with dapsone-induced hypersensitivity syndrome [68], HLA-B $* 1502$ with phenytoin-induced SJS/TEN $[59,70]$, HLA-B $* 3505$ and HLA-BRB1*0101 with nevirapineinduced cutaneous ADRs [74, 84], HLA-B*5701 with 
TABLE 2: Genetic associations of HLA alleles with severe ADRs.

\begin{tabular}{|c|c|c|c|c|}
\hline Drug & HLA allele & $\mathrm{ADR}$ & Ethnic population & Reference \\
\hline Abacavir & $\mathrm{B} * 5701$ & HSS & Caucasian & {$[51,52]$} \\
\hline Allopurinol & $\mathrm{B} * 5801$ & SJS/TEN/HSS & $\begin{array}{c}\text { Han Chinese, Thai, Japanese, } \\
\text { European }\end{array}$ & {$[53-56]$} \\
\hline Aminopenicillins & $\mathrm{A} * 2, \mathrm{DRw} 52$ & DHS & Italian & {$[57]$} \\
\hline Amoxicillin-clavulanate & $\begin{array}{c}\mathrm{A} * 0201 \\
\mathrm{DQB} 1 * 0602\end{array}$ & DILI & Caucasian & {$[58]$} \\
\hline Carbamazepine & $\begin{array}{l}\mathrm{B} * 1502 \\
\mathrm{~B} * 1511 \\
\mathrm{~B} * 5901 \\
\mathrm{~A} * 3101\end{array}$ & SJS/TEN & $\begin{array}{c}\text { Han Chinese, Thai, Indian } \\
\text { Japanese } \\
\text { Japanese } \\
\text { Han Chinese, Japanese, } \\
\text { European }\end{array}$ & $\begin{array}{c}{[59-62]} \\
{[63]} \\
{[64]} \\
{[65-67]}\end{array}$ \\
\hline Dapsone & $\mathrm{B} * 1301$ & HSS & Han Chinese & {$[68]$} \\
\hline Flucloxacillin & $\mathrm{B} * 5701$ & DILI & Caucasian & {$[69]$} \\
\hline Lamotrigine & $\begin{array}{c}\mathrm{B} * 1502, \mathrm{~B} * 38 \\
\mathrm{~B} * 5801, \mathrm{~A} * 6801, \\
\mathrm{Cw} * 0718 \\
\mathrm{DQB} 1 * 0609 \\
\mathrm{DRB} 1 * 1301 \\
\end{array}$ & SJS/TEN & $\begin{array}{c}\text { Han Chinese } \\
\text { European }\end{array}$ & {$[55,70,71]$} \\
\hline Lumiracoxib & $\begin{array}{c}\mathrm{DRB} 1 * 1501 \\
\mathrm{DQB} 1 * 0602 \\
\mathrm{DRB} 5 * 0101 \\
\mathrm{DQA} 1 * 0102\end{array}$ & DILI & Multiple populations & {$[72]$} \\
\hline Methazolamide & $\mathrm{B} * 5901, \mathrm{CW} * 0102$ & SIS/TEN & Korean, Japanese & {$[73]$} \\
\hline Nevirapine & $\begin{array}{c}\mathrm{B} * 3505 \\
\mathrm{DRB} 1 * 0101\end{array}$ & $\begin{array}{l}\text { DHS } \\
\text { DHS }\end{array}$ & $\begin{array}{c}\text { Thai } \\
\text { Australian }\end{array}$ & $\begin{array}{l}{[74]} \\
{[75]}\end{array}$ \\
\hline Oxicam & $\mathrm{B} * 73, \mathrm{~A} * 2, \mathrm{~B} * 12$ & SIS/TEN & European & {$[55,71]$} \\
\hline Oxcarbazepine & $\mathrm{B} * 1502$ & SJS/TEN & Han Chinese & {$[70]$} \\
\hline Phenytoin & $\mathrm{B} * 1502$ & SJS/TEN & Han Chinese, Thai & {$[59,70]$} \\
\hline Sulfamethoxazole & $\mathrm{B} * 38$ & SJS/TEN & European & {$[55]$} \\
\hline
\end{tabular}

HLA: human leukocyte antigen; HSS: hypersensitivity syndrome; SJS/TEN: Stevens-Johnson syndrome/toxic epidermal necrolysis; DHS: delayed-type hypersensitivity reaction; DILI: drug-induced liver injury.

flucloxacillin-induced hepatitis [69], HLA-DPB1*0301 with aspirin-induced asthma [85], and HLA-DQA1*0201 with lapatinib-induced hepatotoxicity [86].

\section{Miscellaneous}

In addition to those mentioned above, genetic variations of many genes that are unrelated to pharmacokinetics/pharmacodynamics and HLA-restricted immune responses have been found to be associated with drug toxicity. These include, but not limited to, various cytokine gene promoters [87-89], epidermal growth factor receptor (EGFR) [90, 91], Fc gamma receptor [92], and microRNAs [93].

\section{Conclusions}

A decade has passed since the completion of the human genome project. During this period, human genetic research has revealed that the genetic backgrounds between the individuals can contribute to differences in the susceptibility to various ADRs. Thousands of genetic variations that are associated with drug safety and toxicity have been identified, many of which have shown high accuracy at predicting drug responses and adverse events. However, the molecular mechanisms through which these biomarkers influence disease risk and/or phenotypic expression still need to be further elucidated. More importantly, to determine which patients will benefit or suffer from a particular drug, the major challenge lies in translating the findings into clinical practice, which perceivably is a key component of the advancement to "personalized medicine."

\section{Conflict of Interests}

The authors declare that there is no conflict of interests regarding the publication of this paper. 


\section{Acknowledgments}

This work was supported by Research Grants from the National Science Council, Taiwan (NSC98-2320-B-010-002MY3, NSC98-2314-B-182A-027-MY3，NSC101-2320-B-010072-MY3, NSC101-2321-B-010-027, NSC101-2628-B-182-001MY3, NSC101-2321-B-182-008, and NSC102-2314-B-010-014MY3) and the Chang Gung Memorial Hospital (BMRPG290011 and CMRPG-290051 3).

\section{References}

[1] J. Lazarou, B. H. Pomeranz, and P. N. Corey, "Incidence of adverse drug reactions in hospitalized patients: a metaanalysis of prospective studies," Journal of the American Medical Association, vol. 279, no. 15, pp. 1200-1205, 1998.

[2] M. D. Rawlings and J. W. Thompson, "Mechanisms of adverse drug reactions," in Textbook of Adverse Drug Reactions, D. M. Davis, Ed., pp. 18-45, Oxford University Press, Oxford, UK, 1991.

[3] K. E. Lasser, P. D. Allen, S. J. Woolhandler, D. U. Himmelstein, S. M. Wolfe, and D. H. Bor, "Timing of new black box warnings and withdrawals for prescription medications," Journal of the American Medical Association, vol. 287, no. 17, pp. 2215-2220, 2002.

[4] J. A. DiMasi, R. W. Hansen, and H. G. Grabowski, "The price of innovation: bew estimates of drug development costs," Journal of Health Economics, vol. 22, no. 2, pp. 151-185, 2003.

[5] N. Risch and K. Merikangas, "The future of genetic studies of complex human diseases," Science, vol. 273, no. 5281, pp. 15161517, 1996.

[6] J. P. A. Ioannidis, E. E. Ntzani, T. A. Trikalinos, and D. G. Contopoulos-Ioannidis, "Replication validity of genetic association studies," Nature Genetics, vol. 29, no. 3, pp. 306-309, 2001.

[7] J. Schmutz, J. Wheeler, J. Grimwood et al., "Quality assessment of the human genome sequence," Nature, vol. 429, no. 6990, pp. 365-368, 2004.

[8] The International HapMap Consortium, "A haplotype map of the human genome," Nature, vol. 437, no. 7063, pp. 1299-1320, 2005.

[9] J. Hardy and A. Singleton, "Genomewide association studies and human disease," The New England Journal of Medicine, vol. 360, no. 17, pp. 1759-1768, 2009.

[10] A. K. Daly, "Using genome-wide association studies to identify genes important in serious adverse drug reactions," Annual Review of Pharmacology and Toxicology, vol. 52, pp. 21-35, 2012.

[11] H. L. Rehm, "Disease-targeted sequencing: a cornerstone in the clinic," Nature Reviews Genetics, vol. 14, no. 4, pp. 295-300, 2013.

[12] E. T. Cirulli and D. B. Goldstein, "Uncovering the roles of rare variants in common disease through whole-genome sequencing," Nature Reviews Genetics, vol. 11, no. 6, pp. 415-425, 2010.

[13] S. C. Sim, M. Kacevska, and M. Ingelman-Sundberg, "Pharmacogenomics of drug-metabolizing enzymes: a recent update on clinical implications and endogenous effects," The Pharmacogenomics Journal, vol. 13, no. 1, pp. 1-11, 2013.

[14] S. A. Sheweita, "Drug-metabolizing enzymes: mechanisms and functions," Current Drug Metabolism, vol. 1, no. 2, pp. 107-132, 2000.

[15] S. Crettol, N. Petrovic, and M. Murray, "Pharmacogenetics of phase I and phase II drug metabolism," Current Pharmaceutical Design, vol. 16, no. 2, pp. 204-219, 2010.
[16] K. A. Phillips, D. L. Veenstra, E. Oren, J. K. Lee, and W. Sadee, "Potential role of pharmacogenomics in reducing adverse drug reactions: a systematic review," Journal of the American Medical Association, vol. 286, no. 18, pp. 2270-2279, 2001.

[17] N. Božina, V. Bradamante, and M. Lovrić, "Genetic polymorphism of metabolic enzymes P450 (CYP) as a susceptibility factor for drug response, toxicity, and cancer risk," Arhiv za Higijenu Rada i Toksikologiju, vol. 60, no. 2, pp. 217-242, 2009.

[18] B. Wang, J. Wang, S.-Q. Huang, H.-H. Su, and S.-F. Zhou, "Genetic polymorphism of the human cytochrome P450 2C9 gene and its clinical significance," Current Drug Metabolism, vol. 10, no. 7, pp. 781-834, 2009.

[19] M. Stojiljkovic, G. P. Patrinos, and S. Pavlovic, "Clinical applicability of sequence variations in genes related to drug metabolism," Current Drug Metabolism, vol. 12, no. 5, pp. 445454, 2011.

[20] J.-S. Hulot, A. Bura, E. Villard et al., "Cytochrome P450 2C19 loss-of-function polymorphism is a major determinant of clopidogrel responsiveness in healthy subjects," Blood, vol. 108, no. 7, pp. 2244-2247, 2006.

[21] S. C. Sim, L. Nordin, T. M.-L. Andersson et al., "Association between CYP2C19 polymorphism and depressive symptoms," The American Journal of Medical Genetics B, vol. 153, no. 6, pp. 1160-1166, 2010.

[22] D. A. Mrazek, J. M. Biernacka, D. J. O’Kane et al., "CYP2C19 variation and citalopram response," Pharmacogenetics and Genomics, vol. 21, no. 1, pp. 1-9, 2011.

[23] U. M. Zanger, M. Turpeinen, K. Klein, and M. Schwab, "Functional pharmacogenetics/genomics of human cytochromes P450 involved in drug biotransformation," Analytical and Bioanalytical Chemistry, vol. 392, no. 6, pp. 1093-1108, 2008.

[24] Y. Gasche, Y. Daali, M. Fathi et al., "Codeine intoxication associated with ultrarapid CYP2D6 metabolism," The New England Journal of Medicine, vol. 351, no. 27, pp. 2827-2831, 2004.

[25] J. Kirchheiner, K. Nickchen, M. Bauer et al., "Pharmacogenetics of antidepressants and antipsychotics: the contribution of allelic variations to the phenotype of drug response," Molecular Psychiatry, vol. 9, no. 5, pp. 442-473, 2004.

[26] F. Innocenti and M. J. Ratain, "Pharmacogenetics of irinotecan: clinical perspectives on the utility of genotyping," Pharmacogenomics, vol. 7, no. 8, pp. 1211-1221, 2006.

[27] G. Toffoli, E. Cecchin, G. Corona et al., "The role of UGT1A1*28 polymorphism in the pharmacodynamics and pharmacokinetics of irinotecan in patients with metastatic colorectal cancer," Journal of Clinical Oncology, vol. 24, no. 19, pp. 3061-3068, 2006.

[28] M. Eichelbaum, M. Ingelman-Sundberg, and W. E. Evans, "Pharmacogenomics and individualized drug therapy," Annual Review of Medicine, vol. 57, pp. 119-137, 2006.

[29] J. M. Heckmann, E. M. T. Lambson, F. Little, and E. P. Owen, "Thiopurine methyltransferase (TPMT) heterozygosity and enzyme activity as predictive tests for the development of azathioprine-related adverse events," Journal of the Neurological Sciences, vol. 231, no. 1-2, pp. 71-80, 2005.

[30] W. E. Evans, Y. Y. Hon, L. Bomgaars et al., "Preponderance of thiopurine S-methyltransferase deficiency and heterozygosity among patients intolerant to mercaptopurine or azathioprine," Journal of Clinical Oncology, vol. 19, no. 8, pp. 2293-2301, 2001.

[31] E. Sim, "Pharmacogenomics of arylamine N-acetyltransferases-from drug metabolism to drug discovery," Pharmacogenomics, vol. 3, no. 6, pp. 729-731, 2002. 
[32] K. M. Das and R. Dubin, "Clinical pharmacokinetics of sulphasalazine," Clinical Pharmacokinetics, vol. 1, no. 6, pp. 406425, 1976.

[33] L. E. Lemke and C. A. McQueen, "Acetylation and its role in the mutagenicity of the antihypertensive agent hydralazine," Drug Metabolism and Disposition, vol. 23, no. 5, pp. 559-565, 1995.

[34] I. Strandberg, G. Boman, L. Hassler, and F. Sjoqvist, "Acetylator phenotype in patients with hydralazine induced lupoid syndrome," Acta Medica Scandinavica, vol. 200, no. 5, pp. 367-371, 1976.

[35] C. D. Klaassen and L. M. Aleksunes, "Xenobiotic, bile acid, and cholesterol transporters: function and regulation," Pharmacological Reviews, vol. 62, no. 1, pp. 1-96, 2010.

[36] M. K. Degorter, C. Q. Xia, J. J. Yang, and R. B. Kim, "Drug transporters in drug efficacy and toxicity," Annual Review of Pharmacology and Toxicology, vol. 52, pp. 249-273, 2012.

[37] M. Niemi, M. K. Pasanen, and P. J. Neuvonen, "Organic anion transporting polypeptide 1B1: a genetically polymorphic transporter of major importance for hepatic drug uptake," Pharmacological Reviews, vol. 63, no. 1, pp. 157-181, 2011.

[38] E. Link, S. Parish, J. Armitage et al., "SLCO1B1 variants and statin-induced myopathy-a genomewide study," The New England Journal of Medicine, vol. 359, no. 8, pp. 789-799, 2008.

[39] L. R. Brunham, P. J. Lansberg, L. Zhang et al., "Differential effect of the rs4149056 variant in SLCO1B1 on myopathy associated with simvastatin and atorvastatin," Pharmacogenomics Journal, vol. 12, no. 3, pp. 233-237, 2012.

[40] R. A. Wilke, L. B. Ramsey, S. G. Johnson et al., "The clinical pharmacogenomics implementation consortium: CPIC guideline for SLCO1B1 and simvastatin-induced myopathy," Clinical Pharmacology \& Therapeutics, vol. 92, no. 1, pp. 112-117, 2012.

[41] K. K. Filipski, R. H. Mathijssen, T. S. Mikkelsen, A. H. Schinkel, and A. Sparreboom, "Contribution of organic cation transporter 2 (OCT2) to cisplatin-induced nephrotoxicity," Clinical Pharmacology \& Therapeutics, vol. 86, no. 4, pp. 396-402, 2009.

[42] Y.-F. Han, X.-H. Fan, X.-J. Wang et al., "Association of intergenic polymorphism of organic anion transporter 1 and 3 genes with hypertension and blood pressure response to hydrochlorothiazide," The American Journal of Hypertension, vol. 24, no. 3, pp. 340-346, 2011.

[43] M. M. Gottesman and I. Pastan, "Biochemistry of multidrug resistance mediated by the multidrug transporter," Annual Review of Biochemistry, vol. 62, pp. 385-427, 1993.

[44] I. A. Hauser, E. Schaeffeler, S. Gauer et al., "ABCB1 genotype of the donor but not of the recipient is a major risk factor for cyclosporine-related nephrotoxicity after renal transplantation," Journal of the American Society of Nephrology, vol. 16, no. 5, pp. 1501-1511, 2005.

[45] M. Garcia, R. M. Macias, J. J. Cubero, J. Benitez, F. Caravaca, and G. Gervasini, "ABCB1 polymorphisms are associated with cyclosporine-induced nephrotoxicity and gingival hyperplasia in renal transplant recipients," European Journal of Clinical Pharmacology, vol. 69, no. 3, pp. 385-393, 2013.

[46] A. Johne, K. Köpke, T. Gerloff et al., "Modulation of steady-state kinetics of digoxin by haplotypes of the P-glycoprotein MDR1 gene," Clinical Pharmacology \& Therapeutics, vol. 72, no. 5, pp. 584-594, 2002.

[47] B. Chowbay, S. Cumaraswamy, Y. B. Cheung, Q. Zhou, and E. J. D. Lee, "Genetic polymorphisms in MDR1 and CYP3A4 genes in Asians and the influence of MDR1 haplotypes on cyclosporin disposition in heart transplant recipients," Pharmacogenetics, vol. 13, no. 2, pp. 89-95, 2003.
[48] S.-Y. Yi, K.-S. Hong, H.-S. Lim et al., "A variant 2677A allele of the MDR1 gene affects fexofenadine disposition," Clinical Pharmacology \& Therapeutics, vol. 76, no. 5, pp. 418-427, 2004.

[49] S.-K. Low, K. Kiyotani, T. Mushiroda, Y. Daigo, Y. Nakamura, and $\mathrm{H}$. Zembutsu, "Association study of genetic polymorphism in ABCC4 with cyclophosphamide-induced adverse drug reactions in breast cancer patients," Journal of Human Genetics, vol. 54, no. 10, pp. 564-571, 2009.

[50] M. Ansari, G. Sauty, M. Labuda et al., "Polymorphisms in multidrug resistance-associated protein gene 4 is associated with outcome in childhood acute lymphoblastic leukemia," Blood, vol. 114, no. 7, pp. 1383-1386, 2009.

[51] A. M. Martin, D. Nolan, S. Gaudieri et al., "Predisposition to abacavir hypersensitivity conferred by HLA-B ${ }^{*} 5701$ and a haplotypic Hsp70-Hom variant," Proceedings of the National Academy of Sciences of the United States of America, vol. 101, no. 12, pp. 4180-4185, 2004.

[52] S. Mallal, D. Nolan, C. Witt et al., "Association between presence of HLA-B* 5701, HLA-DR7, and HLA-DQ3 and hypersensitivity to HIV-1 reverse-transcriptase inhibitor abacavir," The Lancet, vol. 359, no. 9308, pp. 727-732, 2002.

[53] W. Tassaneeyakul, T. Jantararoungtong, P. Chen et al., "Strong association between HLA-B* 5801 and allopurinol-induced Stevens-Johnson syndrome and toxic epidermal necrolysis in a Thai population," Pharmacogenetics and Genomics, vol. 19, no. 9, pp. 704-709, 2009.

[54] N. Kaniwa, Y. Saito, M. Aihara et al., "HLA-B locus in Japanese patients with anti-epileptics and allopurinol-related StevensJohnson syndrome and toxic epidermal necrolysis," Pharmacogenomics, vol. 9, no. 11, pp. 1617-1622, 2008.

[55] C. Lonjou, N. Borot, P. Sekula et al., "A European study of HLA-B in Stevens-Johnson syndrome and toxic epidermal necrolysis related to five high-risk drugs," Pharmacogenetics and Genomics, vol. 18, no. 2, pp. 99-107, 2008.

[56] S.-L. Hung, W.-H. Chung, L.-B. Liou et al., "HLA-B* 5801 allele as a genetic marker for severe cutaneous adverse reactions caused by allopurinol," Proceedings of the National Academy of Sciences of the United States of America, vol.102, no. 11, pp. 41344139, 2005.

[57] A. Romano, A. de Santis, A. Romito et al., "Delayed hypersensitivity to aminopenicillins is related to major histocompatibility complex genes," Annals of Allergy, Asthma \& Immunology, vol. 80, no. 5, pp. 433-437, 1998.

[58] M. I. Lucena, M. Molokhia, Y. Shen et al., "Susceptibility to amoxicillin-clavulanate-induced liver injury is influenced by multiple HLA class I and II alleles," Gastroenterology, vol. 141, no. 1, pp. 338-347, 2011.

[59] C. Locharernkul, J. Loplumlert, C. Limotai et al., "Carbamazepine and phenytoin induced Stevens-Johnson syndrome is associated with HLA-B* 1502 allele in Thai population," Epilepsia, vol. 49, no. 12, pp. 2087-2091, 2008.

[60] W.-H. Chung, S.-I. Hung, H.-S. Hong et al., "Medical genetics: a marker for Stevens-Johnson syndrome," Nature, vol. 428, no. 6982, p. 486, 2004.

[61] C. B. Man, P. Kwan, L. Baum et al., "Association between HLA-B* 1502 allele and antiepileptic drug-induced cutaneous reactions in Han Chinese," Epilepsia, vol. 48, no. 5, pp. 1015-1018, 2007. 
[62] T. Y. Mehta, L. M. Prajapati, B. Mittal et al., "Association of HLA-B* 1502 allele and carbamazepine-induced StevensJohnson syndrome among Indians," Indian Journal of Dermatology, Venereology and Leprology, vol. 75, no. 6, pp. 579-582, 2009.

[63] N. Kaniwa, Y. Saito, M. Aihara et al., "HLA-B*1511 is a risk factor for carbamazepine-induced Stevens-Johnson syndrome and toxic epidermal necrolysis in Japanese patients," Epilepsia, vol. 51, no. 12, pp. 2461-2465, 2010.

[64] H. Ikeda, Y. Takahashi, E. Yamazaki et al., "HLA class I markers in Japanese patients with carbamazepine-induced cutaneous adverse reactions," Epilepsia, vol. 51, no. 2, pp. 297-300, 2010.

[65] M. McCormack, A. Alfirevic, S. Bourgeois et al., "HLA-A*3101 and carbamazepine-induced hypersensitivity reactions in Europeans," The New England Journal of Medicine, vol. 364, no. 12, pp. 1134-1143, 2011.

[66] S.-I. Hung, W.-H. Chung, S.-H. Jee et al., "Genetic susceptibility to carbamazepine-induced cutaneous adverse drug reactions," Pharmacogenetics and Genomics, vol. 16, no. 4, pp. 297-306, 2006.

[67] H. Niihara, T. Kakamu, Y. Fujita, S. Kaneko, and E. Morita, "HLA-A31 strongly associates with carbamazepine-induced adverse drug reactions but not with carbamazepine-induced lymphocyte proliferation in a Japanese population," The Journal of Dermatology, vol. 39, no. 7, pp. 594-601, 2012.

[68] F. R. Zhang, H. Liu, A. Irwanto et al., "HLA-B"13:01 and the dapsone hypersensitivity syndrome," The New England Journal of Medicine, vol. 369, no. 17, pp. 1620-1628, 2013.

[69] A. K. Daly, P. T. Donaldson, P. Bhatnagar et al., "HLA-B5701 genotype is a major determinant of drug-induced liver injury due to flucloxacillin," Nature Genetics, vol. 41, no. 7, pp. 816-819, 2009.

[70] S.-I. Hung, W.-H. Chung, Z.-S. Liu et al., "Common risk allele in aromatic antiepileptic-drug induced Stevens-Johnson syndrome and toxic epidermal necrolysis in Han Chinese," Pharmacogenomics, vol. 11, no. 3, pp. 349-356, 2010.

[71] G. R. Kazeem, C. Cox, J. Aponte et al., "High-resolution HLA genotyping and severe cutaneous adverse reactions in lamotrigine-treated patients," Pharmacogenetics and Genomics, vol. 19, no. 9, pp. 661-665, 2009.

[72] J. B. Singer, S. Lewitzky, E. Leroy et al., "A genome-wide study identifies HLA alleles associated with lumiracoxib-related liver injury," Nature Genetics, vol. 42, no. 8, pp. 711-714, 2010.

[73] S.-H. Kim, M. Kim, K. W. Lee et al., "HLA-B*5901 is strongly associated with methazolamide-induced Stevens-Johnson syndrome/toxic epidermal necrolysis," Pharmacogenomics, vol. 11, no. 6, pp. 879-884, 2010.

[74] S. Chantarangsu, T. Mushiroda, S. Mahasirimongkol et al., "HLA-B*3505 allele is a strong predictor for nevirapineinduced skin adverse drug reactions in HIV-infected Thai patients," Pharmacogenetics and Genomics, vol. 19, no. 2, pp. 139-146, 2009.

[75] A. M. Martin, D. Nolan, I. James et al., "Predisposition to nevirapine hypersensitivity associated with HLA-DRB1*0101 and abrogated by low CD4 T-cell counts," AIDS, vol. 19, no. 1, pp. 97-99, 2005.

[76] R. Pavlos, S. Mallal, and E. Phillips, "HLA and pharmacogenetics of drug hypersensitivity," Pharmacogenomics, vol. 13, no. 11, pp. 1285-1306, 2012.

[77] L. R. Pohl, H. Satoh, D. D. Christ, and J. G. Kenna, "The immunologic and metabolic basis of drug hypersensitivities,"
Annual Review of Pharmacology and Toxicology, vol. 28, pp. 367-387, 1988.

[78] E. Padovan, T. Bauer, M. M. Tongio, H. Kalbacher, and H. U. Weltzien, "Penicilloyl peptides are recognized as $\mathrm{T}$ cell antigenic determinants in penicillin allergy," European Journal of Immunology, vol. 27, no. 6, pp. 1303-1307, 1997.

[79] W. J. Pichler, "Pharmacological interaction of drugs with antigen-specific immune receptors: the p-i concept," Current Opinion in Allergy and Clinical Immunology, vol. 2, no. 4, pp. 301-305, 2002.

[80] P. T. Illing, J. P. Vivian, N. L. Dudek et al., "Immune selfreactivity triggered by drug-modified HLA-peptide repertoire," Nature, vol. 486, no. 7404, pp. 554-558, 2012.

[81] D. A. Ostrov, B. J. Grant, Y. A. Pompeu et al., "Drug hypersensitivity caused by alteration of the MHC-presented self-peptide repertoire," Proceedings of the National Academy of Sciences of the United States of America, vol. 109, no. 25, pp. 9959-9964, 2012.

[82] M. A. Norcross, S. Luo, L. Lu et al., "Abacavir induces loading of novel self-peptides into HLA-B*57: 01: an autoimmune model for HLA-associated drug hypersensitivity," AIDS, vol. 26, no. 11, pp. F21-F29, 2012.

[83] T. Ozeki, T. Mushiroda, A. Yowang et al., "Genome-wide association study identifies HLA-A* 3101 allele as a genetic risk factor for carbamazepine-induced cutaneous adverse drug reactions in Japanese population," Human Molecular Genetics, vol. 20, no. 5, pp. 1034-1041, 2011.

[84] Z. G. Vitezica, B. Milpied, C. Lonjou et al., "HLA-DRB1*01 associated with cutaneous hypersensitivity induced by nevirapine and efavirenz," AIDS, vol. 22, no. 4, pp. 540-541, 2008.

[85] J. H. Choi, K. W. Lee, H. B. Oh et al., "HLa association in aspirinintolerant asthma: $\mathrm{DPB1}^{*} 0301$ as a strong marker in a Korean population," Journal of Allergy and Clinical Immunology, vol. 113, no. 3, pp. 562-564, 2004.

[86] C. F. Spraggs, L. R. Budde, L. P. Briley et al., "HLA-DQA1* 02:01 is a major risk factor for lapatinib-induced hepatotoxicity in women with advanced breast cancer," Journal of Clinical Oncology, vol. 29, no. 6, pp. 667-673, 2011.

[87] K. Sakamoto, M. Oka, S. Yoshino et al., "Relation between cytokine promoter gene polymorphism and toxicity of 5fluorouracil plus cisplatin chemotherapy," Oncology Reports, vol. 16, no. 2, pp. 381-387, 2006.

[88] H.-J. Park, Y.-M. Ye, G.-Y. Hur, S.-H. Kim, and H.-S. Park, "Association between a TGF $\beta 1$ promoter polymorphism and the phenotype of aspirin-intolerant chronic urticaria in a Korean population," Journal of Clinical Pharmacy and Therapeutics, vol. 33, no. 6, pp. 691-697, 2008.

[89] S.-H. Kim, E.-M. Yang, H.-N. Lee, B.-Y. Cho, Y.-M. Ye, and H.-S. Park, "Combined effect of IL-10 and TGF- $\beta 1$ promoter polymorphisms as a risk factor for aspirin-intolerant asthma and rhinosinusitis," Allergy, vol. 64, no. 8, pp. 1221-1225, 2009.

[90] F. Graziano, A. Ruzzo, F. Loupakis et al., "Pharmacogenetic profiling for cetuximab plus irinotecan therapy in patients with refractory advanced colorectal cancer," Journal of Clinical Oncology, vol. 26, no. 9, pp. 1427-1434, 2008.

[91] E. Giovannetti, P. A. Zucali, G. J. Peters et al., "Association of polymorphisms in AKT1 and EGFR with clinical outcome and toxicity in non-small cell lung cancer patients treated with gefitinib," Molecular Cancer Therapeutics, vol. 9, no. 3, pp. 581$593,2010$. 
[92] K. A. Blum, S.-H. Jung, J. L. Johnson et al., "Serious pulmonary toxicity in patients with Hodgkin's lymphoma with SGN30, gemcitabine, vinorelbine, and liposomal doxorubicin is associated with an Fc $\gamma$ RIIIa-158 V/F polymorphism," Annals of Oncology, vol. 21, no. 11, pp. 2246-2254, 2010.

[93] T. Yokoi and M. Nakajima, "microRNAs as mediators of drug toxicity," Annual Review of Pharmacology and Toxicology, vol. 53, pp. 377-400, 2013. 

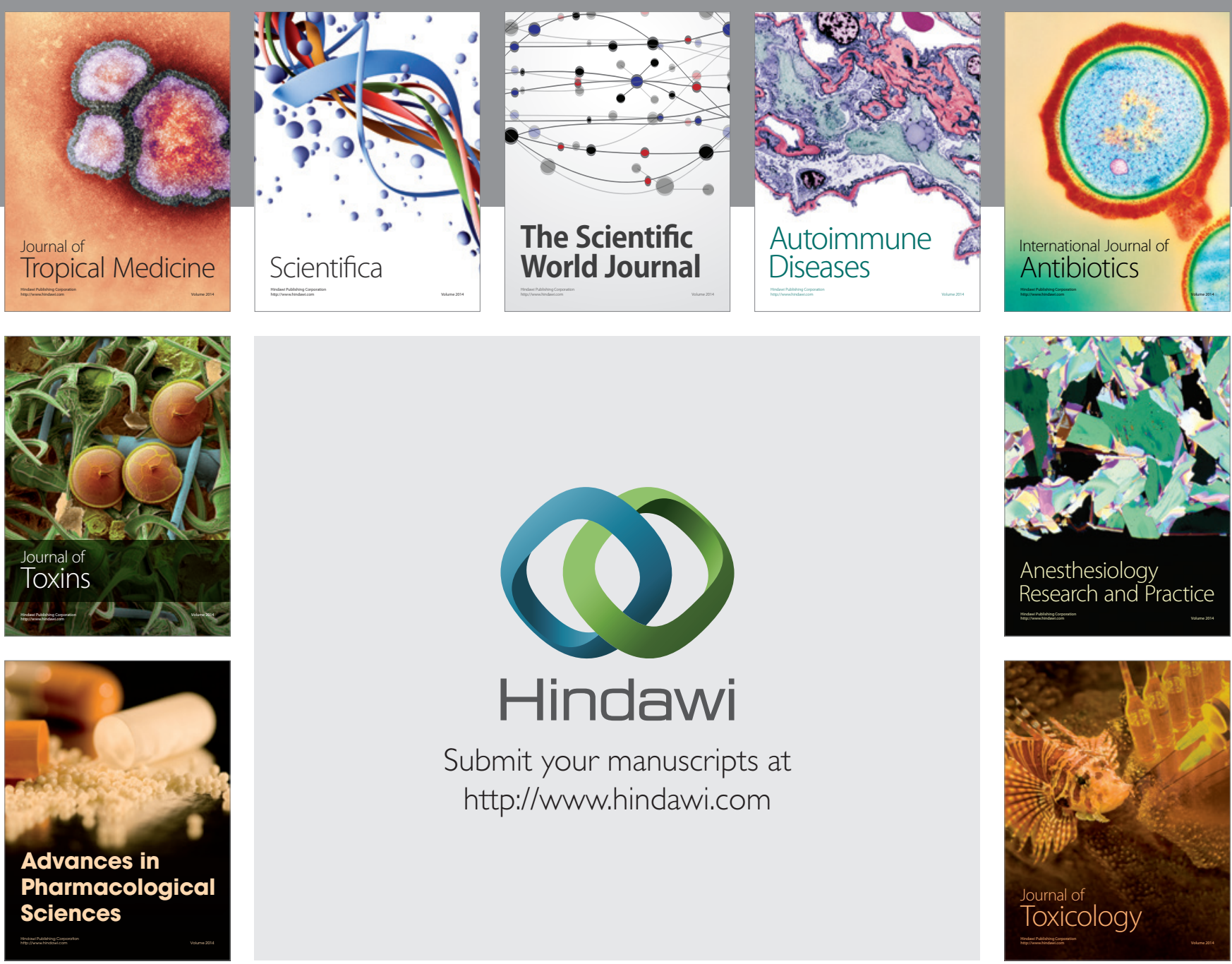

\section{Hindawi}

Submit your manuscripts at

http://www.hindawi.com
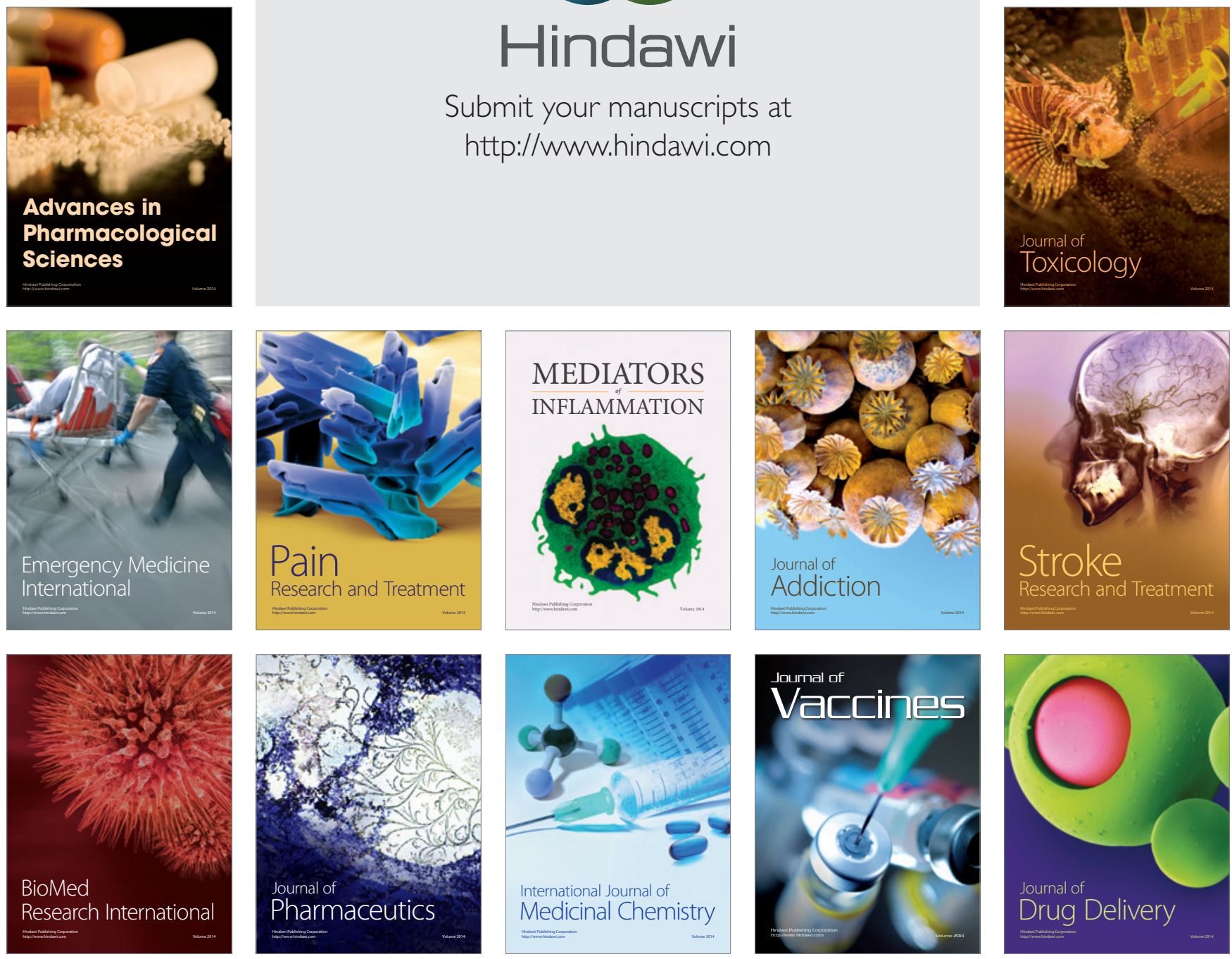